\title{
Antinutriments et propriétés nutritionnelles in vivo de Cochlospermum tinctorium A. Rich. (Bixaceae) chez les jeunes rats (Rattus norvegicus L.)
}

\author{
Collinlaw Joseph NDOUYANG ${ }^{1 *}$, Makhlouf HIMEDA ${ }^{2}$ et \\ Richard Marcel NGUIMBOU ${ }^{3}$
}

\author{
${ }^{1}$ Université des Sciences et de Technologie d'Ati, BP : 20 Ati, Tchad. \\ ${ }^{2}$ Centre National de Nutrition et de Technologie Alimentaire de N'Djaména, BP : 440 N'Djaména, Tchad. \\ ${ }^{3}$ ENSAI, University of Ngaoundére, P.O. Box 455, Ngaoundére, Cameroon. \\ *Auteur correspondant ; E-mail : ndouyang@yahoo.fr; BP : 20 Ati, Tchad; Tél. : (+235)66080163.
}

\section{RESUME}

Cochlospermum tinctorium A. Rich. (Bixaceaee) est une plante répandue dans la bande sahélosoudanienne africaine. Sa racine tubéreuse est utilisée soit comme aliment de soudure et soit comme un organe végétal hépatoprotecteur. Cette racine a été prélevée à Mindaoré/Fianga (Tchad), réduite en poudre et soumise à des analyses chimiques des antinutriments selon des méthodes bien documentées avant d'être testée sur cinq groupes de rats albinos (Rattus norvegicus L). A la fin de l'expérimentation, des analyses biochimiques et hématologiques ainsi que la pesée d'organes ont été effectuées. A un seuil de 5\%, les résultats ont révélé des proportions importantes d'antinutriments dans la racine de $C$. tinctorium. Les corrélations entre ces derniers et les paramètres étudiés et l'évaluation de la masse des différents organes des rats a montré que la consommation de la racine de $C$. tinctorium n'est pas nocive à l'organisme animal mais relance plutôt l'activité des organes vitaux tels que le foie. En définitive, la consommation de $C$. tinctorium a un effet sur le statut pondéral et glycémique et sur le cholestérol total du rat. Néanmoins, il est intéressant d'étudier l'histologie des organes vitaux de l'animal testé.

(C) 2018 International Formulae Group. All rights reserved.

Mots clés: Cochlospermum tinctorium, antinutriments, nutrition, jeune rat.

\section{Antinutrients and in vivo nutritional properties of Cochlospermum tinctorium A. Rich. (Bixaceae) on young male rats (Rattus norvegicus L.)}

\begin{abstract}
Cochlospermum tinctorium A. Rich. (Bixaceaee) is a widespread plant in the african sahelo-soudanian zone. Its tuberous root is used either as famine food, or as medicinal hepatoprotector. This root has been taken in Mindaore/Fianga (Chad), reduced in powder and subjected to chemical analysis of the antinutrients according to well documented methods before testing on five groups of albino rats (Rattus norvegicus $\mathrm{L}$ ). At the end of the experimentation, biochemical and hematological analysis as well as the weighing of organs were carried out. With a threshold of 5\%, the results revealed significant proportions of antinutrients in the root of $C$. tinctorium. The correlations between these antinutrients and the studied parameters, and the evaluation of the mass of the various organs of the rats showed that the consumption of the root of $C$. tinctorium is not harmful
\end{abstract}


with the animal but rather exhibited a revival of the activity of the vital organs such as liver. Ultimately, the consumption of $C$. tinctorium has an effect on the status of the weight and glycemic, and on the total cholesterol of the rat. Nevertheless, it is necessary to study the histology of the vital organs of the tested animal.

(C) 2018 International Formulae Group. All rights reserved.

Keywords: Cochlospermum tinctorium, antinutrients, nutrition, young rat.

\section{INTRODUCTION}

Cochlospermum tinctorium A. Rich. (Bixaceae) a longtemps été utilisé comme un aliment de soudure dans les milieux soudanosahéliens. Les individus végétaux de la strate herbacée jouent ainsi un rôle non négligeable dans la vie des populations tant sur le plan alimentaire que médicinal (Bayaga et al., 2017). La raison vient du changement climatique qui oblige les populations de ces milieux sociobiogéographiques à rechercher des sources alimentaires autres que celles conventionnelles. L'usage repose essentiellement sur la composition chimique du végétal (Muanda et al., 2011; Kouakou et al., 2015), mais justifié par la distribution des composés d'intérêt (Gueye et al., 2012 ). Comme bien d'autres organes végétaux, la racine tubéreuse de $C$. tinctorium n'est pas utilisée seulement comme aliment mais aussi comme organe végétal médicinal (Inngjerdingen et al., 2004 ; Song et al., 2011 ; Zhou et Qu, 2011). Cet usage dépend de la composition chimique des constituants de cette racine. Les composés retrouvés sont : les acides gallique et ellagique, des flavonoïdes (7,3'-diméthyldihydroquercétine; 5,4'diméthylquercetine), des appocaroténoïdes (cochloxanthine, dihydrocochloxanthine) des acetogénines

(cochlospermines, cochlospermatines, cochlospermatinine-type) (Ahmed et al., 2011 ; Musa et al., 2012). En fait, la présence du dihydrokaempferol-3-O- $\beta$ (6"-galloyl)-glucopyranoside a été mentionnée pour la première fois chez $C$. tinctorium par Solon et al. (2012) qui attribuent les effets antifectieux de l'extrait à l'acide gallique. Cette composition chimique est très voisine de l'espèce apparentée, C. planchonii (Bixaceae). Aussi, l'analyse phytochimique des extraits de l'écorce et du bois de la racine de $C$. vitifolium montre plusieurs composés bioactifs rencontrés chez la majorité des espèces du genre Cochlospermum. Parmi ces composés, citons l'acide gallique, les triacylbenzènes ou cochlospermines, le $\beta$-sitostérol et le stigmastérol et leurs D-glucosides (De Almeida et al., 2005). Ayant le même squelette, ces principales cochlospermines, allant de A à D et obtenues par HPLC (High Pressurized Liquid Chromatography), ont des structures symétriques benzéniques triacylées et elles diffèrent entre elles par la longueur des substituants acyl. Par ailleurs, un inhibiteur cristallin d'enzymes P-450, l'aminopyrine-N-déméthylase et l'anilinehydroxylase, a été identifié chez $C$. planchonii (0,3\% MS de rhizomes); il s'agit du formate de zinc qui œuvre comme agent hépatoprotecteur dans le traitement de la jaunisse (Inngjerdingen et al., 2004 ; Willcox et al., 2011; Musa, 2012). La plupart des espèces du genre Cochlopermum sont de plus en plus utilisées pour leur efficacité biologique, à savoir un pouvoir antihyperglycémiant chez Cochlospermum vitifolium (Ortiz-Andrade et al., 2009) ou un pouvoir anti-diabétique chez Cochlospermum planchonii (Yakubu et al., 2010) ou encore un pouvoir d'accroître la spermatogenèse chez Cochlospermum planchonii (Abu et al., 2012), et chez Cochlospermum religiosum (Sasikala et Savithramma, 2012) qui synthétise de nanoparticles d'argent aux propriétés antimicrobiennes ou pouvoirs biocides (ICON, 2008; Hansen, 2009). Les ions $\mathrm{Ag}^{+}$ présentent un large spectre d'action, aussi bien contre les bactéries à Gram positif et à Gram négatif, que les levures, les champignons ou les virus. Ainsi, Feng et al. (2000) ont observé qu'en présence d' $\mathrm{AgNO}_{3}(10 \mu \mathrm{g} / \mathrm{mL})$, chez $E$. coli, la réplication d'ADN et la multiplication 
de la bactérie sont bloquées, la membrane cytoplasmique et la paroi ne sont plus solidaires, mais détériorées (présence de trous) ; l'activité enzymatique respiratoire (par exemple NADH déshydrogénase) est inhibée par interaction des ions métalliques avec les groupements thiols présents notamment dans les protéines. Comme l'indique leur appartenance à la même famille des Bixaceae, les différentes cochlospermines montrent que C. tinctorium et $C$. planchonii ont des génétiques apparentées quand, en général, on considère les individus végétaux dans les parcs agroforestiers des terroirs soudanosahéliens (Morou et al., 2016). Pour bénéficier de ces bienfaits de la racine de $C$. tinctorium, il apparaît intéressant de tester l'usage alimentaire de cette racine et avoir une orientation pratique à portée de main.

\section{MATERIEL ET METHODES}

\section{Echantillonnage du matériel biologique}

Le matériel biologique est constitué de la racine de C. tinctorium A. Rich. (Bixaceae), une plante non-conventionnelle récoltée au Tchad à Mindaoré par Fianga (Région du Mayo-Kebbi Est) où $C$. tinctorium est appelé «Belyewn» par le groupe ethnique Tupuri. Les échantillons ainsi collectés ont été emballés sans nettoyage dans des sacs aérés et acheminés au Laboratoire de Biophysique, Biochimie Alimentaires et Nutrition de l'ENSAI (Université de Ngaoundéré au Cameroun) dans les 48 heures qui ont suivi la récolte. Au laboratoire, les racines ont été débarrassées de la terre et lavées abondamment avec de l'eau de robinet puis laissées au repos pour l'évaporation et l'égouttage total de l'eau de nettoyage.

\section{Préparation des poudres alimentaires}

La production des farines de $C$. tinctorium a été effectuée à partir de la peau et de la matrice interne charnue de la racine (Planche 1). Après dépelliculage, la peau a été découpée en morceaux et séchée à $50{ }^{\circ} \mathrm{C}$ jusqu'à une masse constante (en 18 heures). Cette masse a été broyée pour obtenir la farine de la peau de C. tinctorium. Quant à sa partie intérieure charnue ou chair de la racine, elle a été râpée avant d'être séchée à $50{ }^{\circ} \mathrm{C}$, puis broyée en poudre pour l'obtention de sa farine. Chaque opération de mouture a été effectuée à l'aide d'un broyeur à marteau (model 242, Japan). Ces deux opérations sont résumées par la Figure 1. Les farines ainsi produites ont été scellées dans des sacs en polyéthylène et conservées à $4{ }^{\circ} \mathrm{C}$ jusqu'à utilisation.

\section{Analyses chimiques des poudres}

Les deux poudres alimentaires ont été analysées chimiquement avant d'être testées in vivo sur des jeunes rats albinos (Rattus norvegicus L.). Il s'agit ici des facteurs antinutritionnels analysés par des méthodes bien documentées: oxalates (Day et Underwood, 1986), phytates (Gao et al., 2007), tannins et alcaloïdes (Makkar et al., 2007) et flavonoïdes (Siddiq et al., 2010). Ces méthodes sont décrites dans une de nos publications précédentes (Ndouyang et al., 2015).

\section{Production des rats et dispositif expérimental \\ L'étude a été conduite sur 36 rats} albinos issus de souche wistar Rattus norvegicus (Wistar Institute of Washington, USA), au Département des Sciences Alimentaires et Nutrition de l'ENSAI, Université de Ngaoundéré, Cameroun. Les animaux expérimentaux ont été obtenus par croisement des parents albinos.

Les animaux étaient des jeunes rats âgés d'un mois au début de l'expérience et leurs poids variaient de $50-55 \mathrm{~g}$. Les rats ont été répartis en 5 lots de 6 rats mâles. Les animaux issus d'une même mère ont été répartis dans des lots différents. Chaque animal a été isolé dans une cage à l'animalerie annexée au Laboratoire de Biophysique, de Biochimie Alimentaire et Nutrition. La température oscillait entre 18 et $25{ }^{\circ} \mathrm{C}$. L'expérimentation a duré en moyenne 6 semaines. Durant ce temps, les animaux ont été soumis à deux régimes, un apéritif et un 
aliment isoénergétique. Constitué de la farine de poisson, du manioc, de $C$. tinctorium, un apéritif de 1-1,5 g/rat a été distribué chaque matin aux animaux avant l'aliment isoénergétique. L'apéritif était composé de $20 \%$ de poisson et de $80 \%$ de manioc. Pendant l'expérimentation, les groupes se distinguaient par le degré de substitution de la fécule de manioc par la farine de C. tinctorium (50\% peau $+50 \%$ chair). En fait, groupe 1 ou témoin : aucun apéritif; groupe 2 ou porteur : aucune substitution; groupe CT1 : $33 \%$ de $C$. tinctorium; groupe CT2: $66 \%$ de $C$. tinctorium groupe CT3: $100 \%$ de $C$. tinctorium (sans fécule de manioc). Quant à l'aliment isoénergétique, sa composition se présente comme suit: $55 \%$ de maïs entier; $10 \%$ de poisson; $10 \%$ de blé; $20 \%$ d'arachide; $0,5 \%$ de sel de cuisine et $4,5 \%$ de lait en poudre.

\section{Mesure de la prise alimentaire et des poids}

Les restes d'apéritif et d'aliment isoénergétique ont été collectés et quantifiés par rat pour en déduire la quantité exacte consommée de chaque aliment. Aussi, les rats ont été pesés dès le début de l'expérimentation, ensuite tous les 7 jours jusqu'à la fin des six semaines d'étude. Les poids initial et final des rats ont été rapportés ainsi que la prise alimentaire quotidienne (apéritif et aliment isoénergétique) quantifiées par semaine. En fait, l'aliment isoénergétique était présenté aux animaux après la consommation des apéritifs et était laissé à leur disposition toute la journée pour être retiré en fin de journée. La consommation de l'aliment isoénergétique et celle de l'apéritif ont été évaluées à raison d'une fois par semaine. Mais l'eau a été donnée aux animaux le matin et le soir sans évaluation. Les températures quotidiennes variaient de 18-25 ${ }^{\circ} \mathrm{C}$.

Les paramètres suivants ont été déterminés (Kouadio et al., 2012) :

- Le gain de poids (GP), exprimé en grammes (g). Il représente la différence entre le poids final $\mathrm{Pf}$ et le poids initial $\mathrm{Pi}$ des animaux. Pour obtenir le gain de poids quotidien (GPQ) en $\mathrm{g} / \mathrm{j}$, la valeur calculée en $\mathrm{g}$ a été divisée par la durée (en jours) de l'expérience. Le gain de poids permet l'évaluation des performances de croissance. Le gain de poids quotidien a été calculé comme suit :

$$
\mathrm{GPQ}=\frac{\mathrm{P}_{\mathrm{f}}(\mathrm{g})-\mathrm{P}_{\mathrm{i}}(\mathrm{g})}{\text { Nombre de jours d'expérimentation }}
$$

L'Indice de Consommation (IC) : il est utilisé par les zootechniciens pour l'évaluation de l'efficacité alimentaire. Les aliments ont été pesés et distribués quotidiennement. Pour chaque groupe, les quantités consommées ont été estimées par jour en faisant la différence entre les quantités distribuées et les quantités restantes à la fin de la journée. L'IC peut être exprimé en fonction du temps et en pourcentage (\%). A l'aide des données relevées, l'indice de consommation (IC) a été calculé en faisant le rapport entre la quantité d'aliments consommée pendant un jour ( $\mathrm{g}$ ) et le gain de poids quotidien $(\mathrm{g})$ :

IC

$$
=\frac{\text { Aliment consommé pendant un temps } \mathrm{t}}{\text { Gain de poids pendant } \mathrm{t}}
$$

- Le coefficient d'efficacité alimentaire (CEA) et le ratio de conversion alimentaire (RCA) : ils traduisent le rendement avec lequel l'aliment est assimilé. Ils ont été déterminés selon la méthode de Dada (2012).. Le CEA a été calculé en faisant le rapport entre le gain de poids (g) et la quantité de matières sèches totales ingérées (MSTI) (g) :

$$
\mathrm{CEA}=\frac{\mathrm{GP}}{\mathrm{MSTI}} * 100
$$

ET le RCA a été calculé par la formule :

$$
\begin{aligned}
& \mathrm{RCA}=\frac{1}{\mathrm{CEA}} * 100=\frac{\mathrm{MSTI}}{\mathrm{GP}} * 100 \\
& \text { Le taux de croissance spécifique }
\end{aligned}
$$
(TCS) a été calculé par la formule suivante (Dada, 2012) :

$$
\begin{gathered}
\operatorname{TCS}\left(\% \cdot \mathrm{j}^{-1}\right)=\frac{\ln \left(\mathrm{P}_{\mathrm{t}}\right)-\ln \left(\mathrm{P}_{0}\right)}{\mathrm{t}} * 100 \\
\text { où: } \quad \boldsymbol{P}_{\boldsymbol{t}} \text { et } \boldsymbol{P}_{\boldsymbol{0}} \quad \text { représentent }
\end{gathered}
$$
respectivement les poids corporels final et initial des rats, et $t$ représente la durée de l'expérimentation. 


\section{Sacrifices des animaux et prélèvements}

$\mathrm{Au}$ terme de l'expérimentation, les animaux ont été sacrifiés sous anesthésie au chloroforme. Le sang a été prélevé dans des tubes à héparine par incision de la veine jugulaire pour les analyses hématologiques et les analyses biochimiques portées sur la glycémie à jeun, l'alanine aminotransférase (ALAT), l'aspartate aminotransférase (ASAT), la gamma-glutamyltransférase $(\gamma$ GT), Cholestérol total et HDL (High Density Liporotein) et ses fractions LDL, des triglycérides (TG), des protéines plasmatiques. Aussi, les organes suivants ont été prélevés et pesés sur une balance d'une précision à $10^{-2}$ près (Gibertini, EU-C 502, Italy) : cerveau, cœur, poumons, estomac, foie, reins. Par ailleurs, les fèces ont été collectées pour le dosage des protéines et lipides fécaux.

\section{Analyses hématologiques et biochimiques}

La numération sanguine, la glycémie

à jeun, trois enzymes (ALAT, ASAT, $\gamma$-GT), les triglycérides, le cholestérol total, les Cholestérols HDL et LDL, les protéines plasmatiques ainsi que la glycémie à jeun, la numération sanguine, les protéines et lipides fécaux ont été dosés selon des méthodes bien documentées telles que rapportés précédemment dans l'une de nos publications (Ndouyang et al., 2014).

\section{Analyses statistiques}

Les moyennes et les écartypes des 6 valeurs individuelles ont été calculés. L'analyse de variance simple a été effectuée pour évaluer les effets de la diète sur le sang et les paramètres des fèces. Quand l'effet est significatif $(p<0,05)$, le test multiple de Duncan (Duncan multiple test range) est réalisé pour comparer les moyennes deux à deux affectées de leurs respectifs. Le logiciel Statgraphics Plus 5.0 a été utilisé pour les analyses statistiques faites à un niveau de signification $\alpha=0,05$.

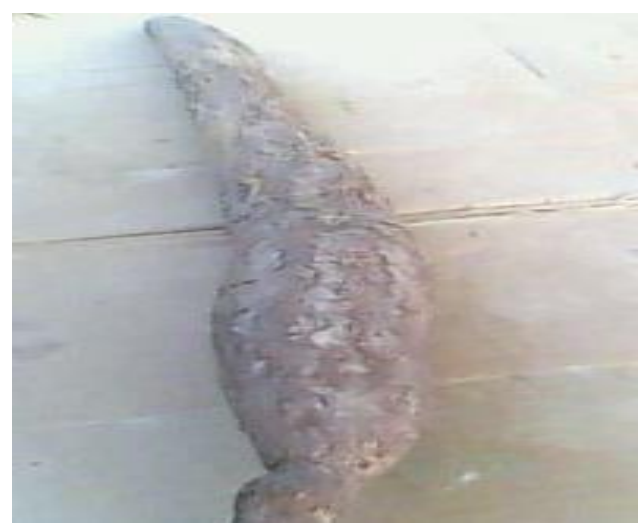

1A: Racine de C. tinctorium

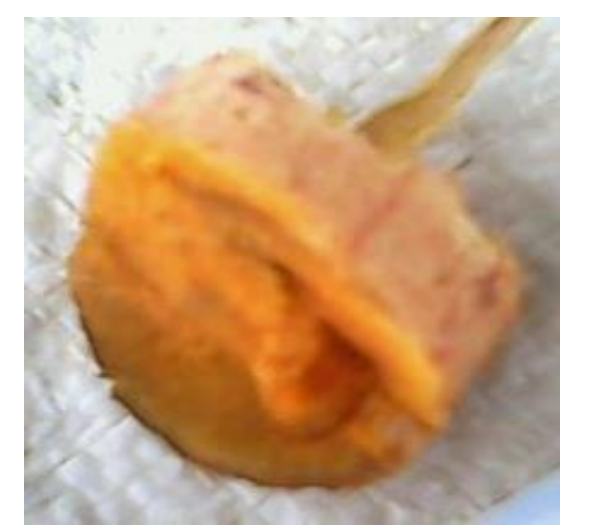

1B: Jeune racine épluchée de $C$. tinctorium

Planche 1: Racine de C. tinctorium. 


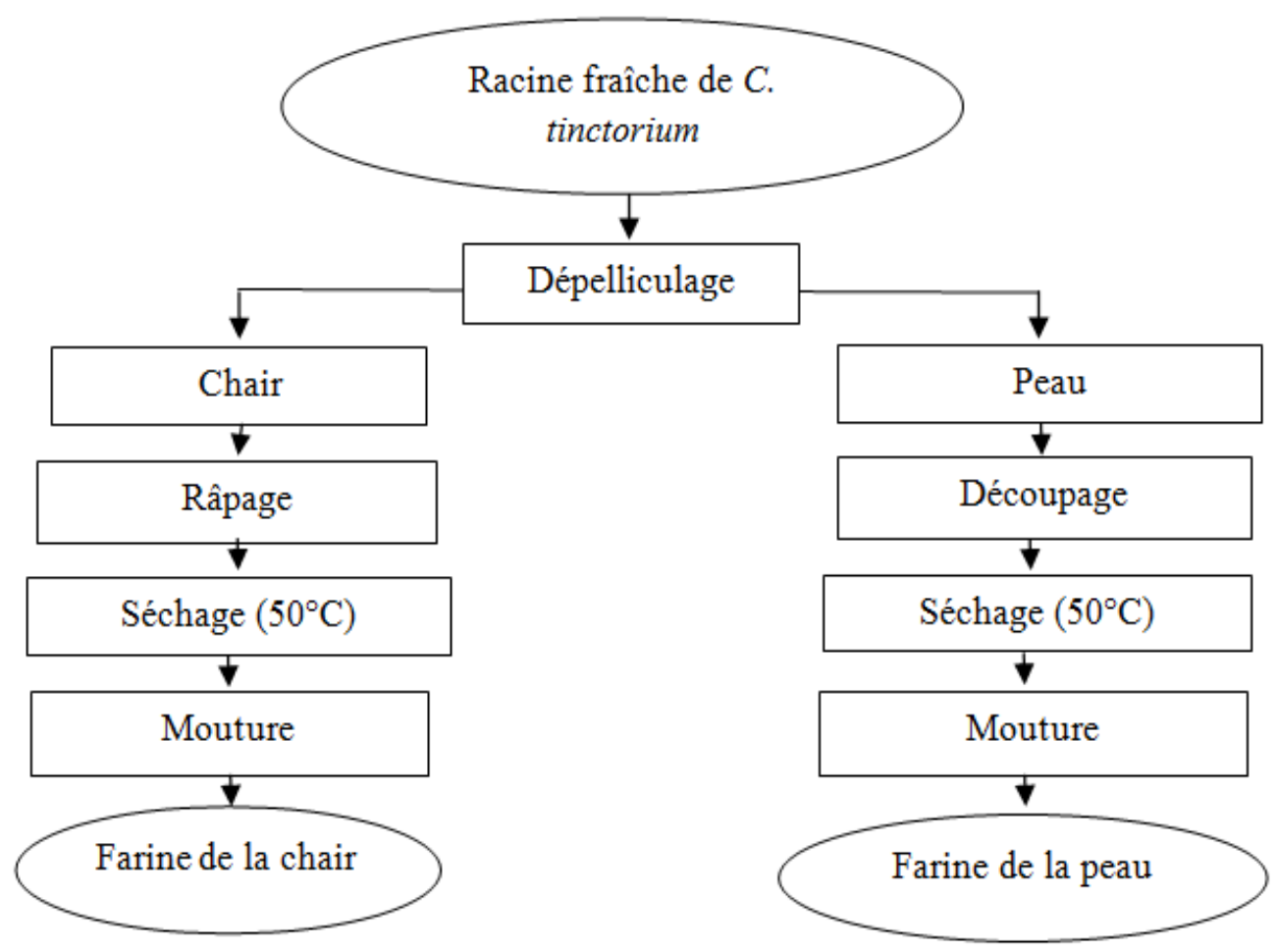

Figure 1 : Diagramme de préparation de la farine de la peau et de la farine de la chair de la racine de C. tinctorium.

\section{RESULTATS}

\section{Consommation des farines de $\boldsymbol{C}$. tinctorium}

Le degré de consommation ou d'acceptabilité des apéritifs a varié d'une farine à l'autre selon qu'on considère les farines de $C$. tinctorium. Ainsi, des différences statistiques $(\mathrm{p}<0,01)$ ont été relevées à la fois pour les farines de $C$. tinctorium.

Avec l'apéritif de $C$. tinctorium (Tableau 1), la consommation des apéritifs a variable selon les groupes tests : $100 \%$ dans le groupe manioc ou groupe 1 . Aussi dans le groupe2, la consommation de l'apéritif à base de C. tinctorium (CT) a été de $100 \%$ contre $91,30 \pm 2,44 \%$ dans le groupe 3 et $88,48 \pm 2,13 \%$ dans le groupe 4 , soit respectivement $\quad 0,23 \quad$ g.MS/rat $/ \mathrm{j} ; \quad 0,42$ g.MS/rat/j et 0,56 g.MS/rat/j.

Ainsi, on note que la prise de la farine de $C$. tinctorium a été appréciable permettant donc une consommation régulière. Il se dégage de cette étude comparative que $C$. tinctorium a bien été pris par le rat à raison de $100-88,48 \%(0,23$ à 0,56 g.MS/rat/j) selon l'augmentation de la dose.

\section{Digestion des farines de $\boldsymbol{C}$. tinctorium}

L'assimilation de la farine de $C$. tinctorium a eu des effets similaires sur la performance nutritionnelle des rats. En fait, des différences significatives $(\mathrm{p}<0,05)$ ont été relevées au niveau des paramètres de digestion des poudres de $C$. tinctorium (CT). Les faits suivants ont été relevés :

(1) La teneur en protéines fécales a été statistiquement la même $(p=0,3716)$ chez les groupes CT1, CT2 et CT3 ayant consommé de C. tinctorium, soit respectivement $20,92 \pm 2,40 \% ; 26,38 \pm 1,91 \%$ et $26,54 \pm 2,16 \%$ contre $24,18 \pm 2,62 \%$ chez le groupe témoin sans apéritif.

(2) La teneur en lipides fécaux a été élevée chez le groupe CT3, soit $10,74 \pm 0,81 \%$ contre 
$8,41 \pm 1,00 \%$ chez le groupe témoin sans apéritif.

(3) Les teneurs en cendres fécales ont été faibles $(\mathrm{p}<0,05)$ chez les groupes dont les apéritifs ont été à base de $C$. tinctorium $(9,87 \pm 0,15 \%$ à $11,30 \pm 0,75 \%)$ contre celle du groupe témoin $(13,15 \pm 0,49 \%)$.

\section{Teneurs en facteurs antinutritionnels dans les apéritifs}

Les facteurs antinutritionnels (FANs) ou antinutriments mis en exergue dans la farine de $C$. tinctorium sont présentés dans le Tableau 2. Dans chacun des groupes tests, la consommation des FANs est croissante selon la consommation des apéritifs. L'influence de chaque antinutriment sera donc mise en évidence et discutée par une étude de corrélation suivant une analyse en composante principale (ACP) effectuée dans ce travail.

\section{Paramètres hématologiques et poids des organes des rats testés}

Les résultats des analyses statiques de paramètres hématologiques chez les rats testés aux farines de $C$. tinctorium sont présentés dans le Tableau 3. Il ressort de ces analyses qu'il n'existe aucune différence statistique significative $(p>0,05)$ entre les différents groupes de rats au point de vue hématologiques sans exception. Cela a révélé que, selon les prises des apéritifs effectuées par les rats, ces deux sources alimentaires n'ont pas affecté les éléments sanguins. Ces résultats démontrent de l'absence de perturbation au niveau des éléments sanguins, qu'il s'agisse des globules rouges et blancs.

L'absence de différence statistique significative $(p>0,05)$ a été aussi observée au sein des groupes expérimentaux pour les six organes pesés (cœur, poumons, estomac, foie, reins et cerveau) après sacrifice des rats (Figure 2). Cette réponse atteste que les différents organes n'ont pas été affectés par la dose prise. Aussi, l'uniformité des poids des organes des rats pour les différents groupes sans exception apparaît démontrer l'innocuité des farines de $C$. tinctorium à la dose prise. Toutefois, il convient de poursuivre la recherche des effets de la farine de $C$. tinctorium en conditions in vivo par l'étude du comportement de trois transaminases.

\section{Paramètres plasmatiques, fécaux et pondéraux des rats}

En fait, l'élimination des lipides fécaux par les rats ayant consommé de la farine de $C$. tinctorium a eu une incidence sur le rapport gain-poids/poids-initial dont la tendance a été très peu à la hausse traduisant un gain de poids relativement faible par rapport au témoin (Figure 3). Egalement, le gain de poids rapporté à la masse du rat (g/rat) et la teneur en protéines plasmatiques baissaient lorsque la teneur en protéines fécales s'élevait, et vice versa. Cela montre que $C$. tinctorium pouvait perturber la croissance du jeune animal s'il était consommé en quantités supérieures à celles mentionnées dans le présent travail. En somme, la chute légère du taux des protéines plasmatiques explique la présence des protéines et des lipides en proportions élevées dans les fèces. Aussi, la glycémie a été affectée par une baisse sensible chez tous les groupes ayant consommé de C. tinctorium (Figure 3).

En effet, l'évolution des poids corporels montre que les rats ayant consommé de $C$. tinctorium ont présenté des poids vifs en-dessous de ceux des groupes témoin et manioc (Référence) mais sans différence statistique significative $(p>0,05)$. C'est ainsi que la croissance a été régulière du début à la fin de l'expérimentation.

\section{Effets de la consommation de $C$. tinctorium sur les paramètres lipidiques}

Le Tableau 3 présente les effets de la consommation de $C$. tinctorium sur les paramètres lipidiques: les Triglycérides, le cholestérol total, les fractions de cholestérol HDL, LDL et VLDL. Selon le test statistique de Duncan $(\mathrm{p}<0,01)$, seul le groupe témoin a présenté des paramètres lipidiques de faibles valeurs par rapport aux quatre autres groupes allant du groupe manioc (groupe 1) au dernier groupe test (groupe 4) qui ont été tous 
identiques d'un paramètres lipides à un autre. Toutefois, le risque d'attaque cardiaque (RAC) a été multiplié par 1,5 chez le manioc et par 2,25 chez les groupes 1 ou par 2 chez le groupe 3, ou encore par 1,75 chez le dernier groupe. Cela montre que les groupes 2,3 et 4 ayant consommé une quantité de C. tinctorium à raison de $0,23 \mathrm{~g} . \mathrm{MS} / \mathrm{rat} / \mathrm{j}, 0,42 \mathrm{~g} . \mathrm{MS} / \mathrm{rat} / \mathrm{j}$ et $0,56 \mathrm{~g} . \mathrm{MS} / \mathrm{rat} / \mathrm{j}$ ont constitué des groupes à risque. Mais ce risque d'attaque cardiaque $\boldsymbol{y}$ décroît avec l'augmentation de la quantité consommée $\boldsymbol{q}$ de $C$. tinctorium selon l'équation $\mathrm{y}=-1,503 \mathrm{q}+2,606 \& \mathrm{R}^{2}=0,992$.

\section{Effets de C. tinctorium sur les transaminases \\ Les résultats des tests de $C$.} tinctorium sur les transaminases (ALAT et
ASAT et GGT) chez le rat sur un prelèvement sanguin à jeun sont présentés dans le Tableau 4. Les analyses statistiques ont révélé de différences significatives $(\mathrm{p}<0,05)$ par rapport au groupe témoin. Seul le CT1 $(0,23$ g.MS/rat/j) a donné un ratio ASAT/ALAT voisin de 1 , tandis que chez les deux derniers groupes, ce ratio a été proche de 2 . Mais les concentrations plasmatiques en $\gamma$-GT ont été inférieures à $5 \mathrm{UI} / \mathrm{L}$ chez tous les groupes, y compris le groupe témoins témoins. Néanmoins, on peut émettre que cette enzyme ne présenterait pas un taux décélable chez le jeune rat. Ainsi, il serait intéressant d'approfondir la connqaissance de l'effet de la consommation de $C$. tinctorium sur cette enzyme.

Tableau 1: Performance nutritionnelle des rats ayant reçu les différents apéritifs de $C$. tinctorium (CT).

\begin{tabular}{|c|c|c|c|c|c|}
\hline \multirow[b]{2}{*}{ Paramètres } & \multirow{2}{*}{$\begin{array}{l}\text { Témoin } \\
\text { (0 apéritif) }\end{array}$} & \multirow{2}{*}{$\begin{array}{c}\text { Porteur } \\
(\text { manioc } 100 \%)\end{array}$} & \multicolumn{3}{|c|}{$\begin{array}{c}\text { Groupes tests à l'apéritif de } C \text {. tinctorium } \\
\text { (g.MS/j) }\end{array}$} \\
\hline & & & CT1 (33\%) & CT2 (66\%) & CT3 $(100 \%)$ \\
\hline Ration (g/rat/j) & $8,46 \pm 0,26^{\mathrm{a}}$ & $8,18 \pm 0,40^{\mathrm{a}}$ & $8,33 \pm 0,24^{\mathrm{a}}$ & $8,42 \pm 0,31^{\mathrm{a}}$ & $7,73 \pm 0,32^{\mathrm{a}}$ \\
\hline Prise d'apéritif (\%) & & $100^{\mathrm{b}}$ & $100^{\mathrm{b}}$ & $91,30 \pm 2,44^{\mathrm{a}}$ & $88,48 \pm 2,13^{\mathrm{a}}$ \\
\hline Apéritif (g.MS.CT/rat/j) & & & $0,23^{\mathrm{a}}$ & $0,42 \pm 0,02^{\mathrm{b}}$ & $0,56 \pm 0,02^{\mathrm{c}}$ \\
\hline CT/ration $(\%)$ & & & $2,74 \pm 0,09^{\mathrm{a}}$ & $5,10 \pm 0,36^{\mathrm{b}}$ & $7,23 \pm 0,29^{\mathrm{c}}$ \\
\hline Cendres fécales $(\%)$ & $13,15 \pm 0,49^{\mathrm{c}}$ & $11,30 \pm 0,44^{\mathrm{b}}$ & $9,87 \pm 0,15^{\mathrm{a}}$ & $10,71 \pm 0,56^{\mathrm{b}}$ & $11,30 \pm 0,75^{\mathrm{b}}$ \\
\hline Poids initial Pi (g) & $38,37 \pm 1,41^{\mathrm{a}}$ & $37,86 \pm 2,05^{\mathrm{a}}$ & $37,66 \pm 3,01^{\mathrm{a}}$ & $36,47 \pm 2,23^{\mathrm{a}}$ & $33,69 \pm 1,34^{\mathrm{a}}$ \\
\hline Poids final Pf (g) & $152,27 \pm 5,96^{\mathrm{a}}$ & $153,32 \pm 8,69^{\mathrm{a}}$ & $146,37 \pm 7,75^{\mathrm{a}}$ & $147,02 \pm 6,95^{\mathrm{a}}$ & $140,40 \pm 7,25^{\mathrm{a}}$ \\
\hline IC (g.aliment/g.p.c.) & $0,08^{\mathrm{a}}$ & $0,07^{\mathrm{a}}$ & $0,08^{\mathrm{a}}$ & $0,08^{\mathrm{a}}$ & $0,07^{\mathrm{a}}$ \\
\hline CEA $(\%)$ & $224,84 \pm 13,96^{\mathrm{a}}$ & $234,14 \pm 7,40^{\mathrm{a}}$ & $216,44 \pm 9,95^{\mathrm{a}}$ & $212,91 \pm 1024^{\mathrm{a}}$ & $229,32 \pm 10,10^{\mathrm{a}}$ \\
\hline $\mathrm{RCA}(\%)$ & $45,34 \pm 2,28^{\mathrm{a}}$ & $42,91 \pm 1,26^{\mathrm{a}}$ & $46,67 \pm 206^{\mathrm{a}}$ & $47,52 \pm 2,32^{\mathrm{a}}$ & $43,39 \pm 1,77^{\mathrm{a}}$ \\
\hline $\operatorname{TCS}(\% / \mathrm{j})$ & $3,28 \pm 0,16^{\mathrm{a}}$ & $3,33 \pm 0,16^{\mathrm{a}}$ & $3,25 \pm 0,20^{\mathrm{a}}$ & $3,33 \pm 0,16^{\mathrm{a}}$ & $3,39 \pm 0,15^{\mathrm{a}}$ \\
\hline
\end{tabular}


Tableau 2: Teneur en antinutriments des apéritifs de $C$. tinctorium consommé.

\begin{tabular}{lcccccc}
\hline & & \multicolumn{5}{c}{ Facteurs antinutritionnels (mg/100 g.MS) } \\
\cline { 3 - 7 } Groupes* & Apéritif $(\mathbf{g})$ & Oxalates & Phytates & Tannins & Alcalö̈des & Flavonoïdes \\
\hline Témoin $(0)$ & & & & & & \\
Porteur $(0,75)$ & & & & & & \\
Groupe CT1 $(0,23)$ & 0,23 & 2,46 & 3,74 & 5,50 & 0,50 & 1,64 \\
Groupe CT2 $(0,42)$ & 0,42 & 4,59 & 6,98 & 10,25 & 0,93 & 3,06 \\
Groupe CT3 $(0,56)$ & 0,56 & 6,02 & 9,15 & 13,44 & 1,22 & 4,01 \\
\hline *Les valeurs entre parenthèses devant les groupes sont les quantités d'apéritifs effectivement consommées par les rats en \\
g.MS/rat/j.
\end{tabular}

Tableau 3: Effet de C. tinctorium sur le profil lipidique et indices athérogéniques chez le rat.

\begin{tabular}{lccccc}
\hline & & \multicolumn{3}{c}{ Apéritif à base de C. tinctorium $(\mathbf{g . M S} / \mathbf{r a t} / \mathbf{j})$} \\
\cline { 4 - 6 } Paramètres & & & & & \\
lipidiques & Témoin (0) & $($ manioc 0,75) & CT1 $(\mathbf{0 , 2 3})$ & CT2 (0,42) & CT3 (0,56) \\
\hline TG (mg/l) & $748,33 \pm 45,86^{\mathrm{a}}$ & $1078,33 \pm 98,81^{\mathrm{ab}}$ & $1231,67 \pm 44,60^{\mathrm{ab}}$ & $1015,00 \pm 58,35^{\mathrm{ab}}$ & $1146,67 \pm 102,17^{\mathrm{ab}}$ \\
C-total (mg/l) & $480,00 \pm 19,66^{\mathrm{a}}$ & $696,67 \pm 47,87^{\mathrm{ab}}$ & $708,33 \pm 58,33^{\mathrm{ab}}$ & $623,33 \pm 71,07^{\mathrm{ab}}$ & $745,00 \pm 88,19^{\mathrm{ab}}$ \\
C-HDL (mg/l) & $328,33 \pm 28,16^{\mathrm{a}}$ & $333,83 \pm 30,57^{\mathrm{ab}}$ & $288,83 \pm 20,79^{\mathrm{ab}}$ & $263,67 \pm 22,38^{\mathrm{ab}}$ & $304,83 \pm 11,89^{\mathrm{ab}}$ \\
C-LDL (mg/l) & $33,17 \pm 11,76^{\mathrm{a}}$ & $147,17 \pm 43,30^{\mathrm{ab}}$ & $185,50 \pm 60,80^{\mathrm{ab}}$ & $156,67 \pm 65,56^{\mathrm{ab}}$ & $210,83 \pm 80,64^{\mathrm{ab}}$ \\
C-VLDL (mg/l) & $149,67 \pm 9,17^{\mathrm{a}}$ & $215,67 \pm 19,76^{\mathrm{ab}}$ & $246,33 \pm 8,92^{\mathrm{ab}}$ & $203,00 \pm 11,67^{\mathrm{ab}}$ & $229,33 \pm 20,43^{\mathrm{ab}}$ \\
TG/C-HDL & $2,33 \pm 0,16^{\mathrm{a}}$ & $3,37 \pm 0,42^{\mathrm{ab}}$ & $4,41 \pm 0,42^{\mathrm{ab}}$ & $4,04 \pm 0,48^{\mathrm{ab}}$ & $3,77 \pm 0,32^{\mathrm{ab}}$ \\
IA1 & $1,51 \pm 0,12^{\mathrm{a}}$ & $2,15 \pm 0,20^{\mathrm{ab}}$ & $2,57 \pm 0,38^{\mathrm{ab}}$ & $2,41 \pm 0,27^{\mathrm{ab}}$ & $2,46 \pm 0,30^{\mathrm{ab}}$ \\
IA2 & $0,51 \pm 0,12^{\mathrm{a}}$ & $1,15 \pm 0,20^{\mathrm{ab}}$ & $1,57 \pm 0,38^{\mathrm{ab}}$ & $1,41 \pm 0,27^{\mathrm{ab}}$ & $1,46 \pm 0,30^{\mathrm{ab}}$ \\
RAC* (x4) & 1 & 1,5 & 2,25 & 2 & 1,75 \\
\hline
\end{tabular}

Les moyennes de la même ligne portant en exposant des lettres différentes sont significativement différentes selon le test de Duncan $(\alpha=0,05 ; \quad p<0,05)$. IA1=cholestérol-total/cholestérol-HDL, IA2=cholestérol VLDL+LDL/ cholestérol-HDL, *RAC=Risque d'attaque cardiaque.

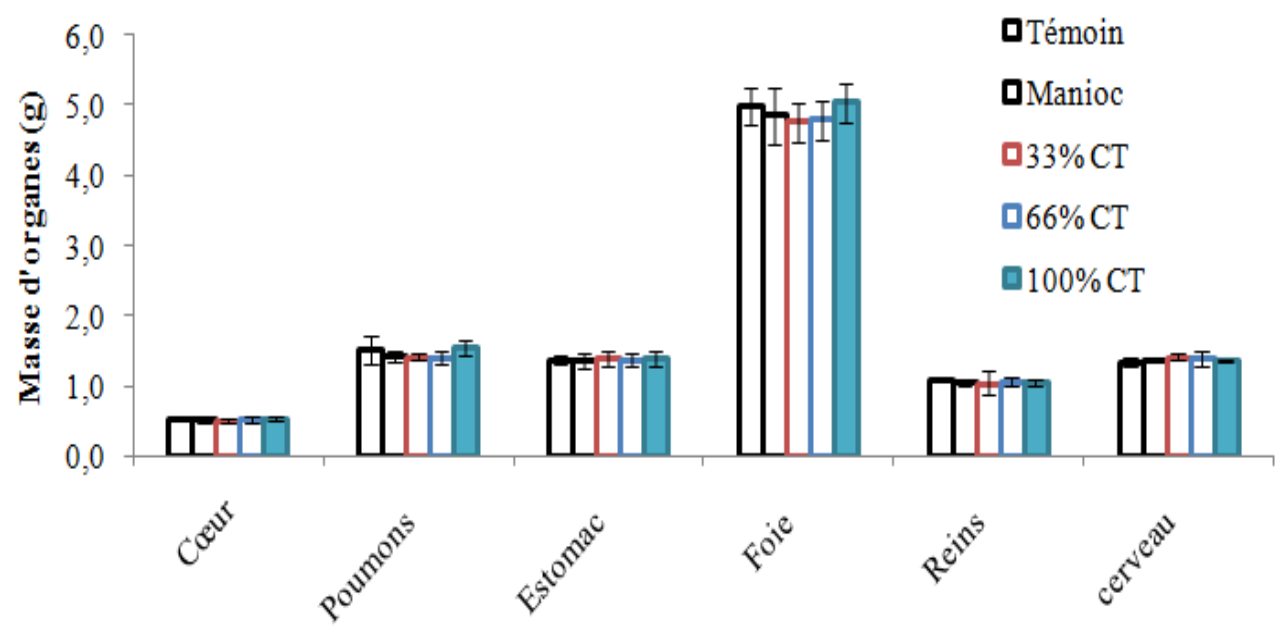

Organes des rats

Figure 2: Poids des organes des rats ayant consommé des apéritifs à base de C. tinctorium. 


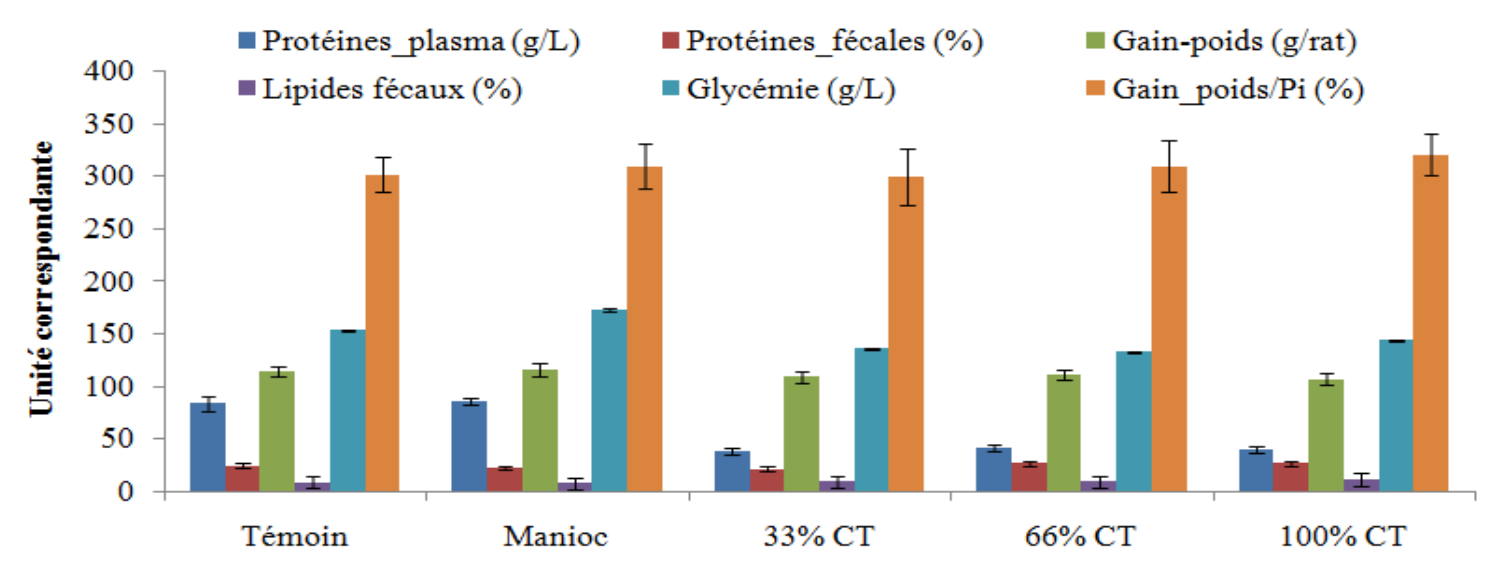

Apéritif de C. tinctorium (g.MS/rat/j)

Figure 3: Paramètres plasmatiques, fécaux et pondéraux des rats en focntion des appéritifs de $C$. tinctorium.

Tableau 4: Concentrations en transaminases plasmatiques chez les rats ayant reçu d'apéritifs de $C$. tinctorium.

\begin{tabular}{lcccc}
\hline Lots de rats & ALAT $(\mathbf{U I} / \mathbf{L})$ & ASAT $(\mathbf{U I} / \mathbf{L})$ & $\gamma$-GT $(\mathbf{U I} / \mathbf{L})$ & ASAT/ALAT \\
\hline Témoin $(0)$ & $52,55 \pm 3,85^{\mathrm{a}}$ & $44,00 \pm 2,00^{\mathrm{a}}$ & $<5$ & $0,84 \pm 0,02^{\mathrm{a}}$ \\
Manioc $(0,75)$ & $53,70 \pm 0,10^{\mathrm{a}}$ & $45,45 \pm 2,05^{\mathrm{a}}$ & $<5$ & $0,85 \pm 0,04^{\mathrm{a}}$ \\
CT1 $(0,23)$ & $63,05 \pm 0,25^{\mathrm{ab}}$ & $86,75 \pm 4,45^{\mathrm{b}}$ & $<5$ & $1,38 \pm 0,08^{\mathrm{b}}$ \\
CT2 $(0,42)$ & $72,75 \pm 0,75^{\mathrm{b}}$ & $120,35 \pm 0,75^{\mathrm{c}}$ & $<5$ & $1,65 \pm 0,01^{\mathrm{c}}$ \\
CT3 $(0,56)$ & $78,60 \pm 3,10^{\mathrm{b}}$ & $131,50 \pm 3,45^{\mathrm{d}}$ & $<5$ & $1,72 \pm 0,11^{\mathrm{c}}$
\end{tabular}

Les moyennes de la même colonne portant en exposant des lettres différentes sont significativement différentes selon le test de Duncan $(\mathrm{p}<0,01)$. ALAT $=$ Alanine AminoTranférase; ASAT $=$ Aspartate AminoTranférase; $\gamma$-GT = Gamma-Glutamyl Transférase. Statistiques. Témoin $=$ sans apéritif; $\mathrm{CT}=\mathrm{C}$. tinctorium.

\section{DISCUSSION}

\section{Profil lipidique des rats}

Chez les rats des groupes CT1, CT2 et CT3 ayant reçu de $C$. tinctorium comme apéritif, les teneurs en cholestérol-LDL et en triglycérides (TG) plasmatiques ont été audessus de celle du groupe témoin sans apéritif. Mais les facteurs de RAC (Risque d'attaque cardiaque) et les indices athérogènes (IA1 et IA2) ont été décroissants chez les groupes ayant consommé de $C$. tinctorium quand les quantités consommées augmentaient. En fait, l'amidon se transforme en graisse sous forme de triglycérides TG avec élévation du taux du cholestérol total. Cela permet de comprendre l'accumulation concomitante des fractions de cholestérol chez le groupe porteur dont l'apéritif a été à base de fécule de manioc. Selon Jeppesen et al. (2001), le risque d'attaque cardiaque (RAC) y est linéairement lié au ratio TG/C-HDL $x$ tel que l'ait: $y=2,406 x-1,973 ; \mathrm{R}^{2}=0,997$. Le ratio $T G / C$ $H D L$ recherché est de 1 correspondant à une plage minimale de RAC. Par contre les RACs deviennent évidents lorsque le ratio TG/CHDL devient très supérieur à 3 (TG/CHDL>3). Dans la présente étude, le RAC est négativement corrélé à la prise de $C$. 
tinctorium. D'après les travaux de (Musa, 2012), la racine de C. tinctorium contient deux principaux caroténoïdes appelés apocaroténoïdes qui sont la cochloxanthine et la dihydrochloxanthine. Egalement, l'effet lipidémiant de $C$. tinctorium utilisé comme aliment a été rapporté par Akinloye et Ayankojo (2011). Sur le plan physiologique, les apocaroténoïdes assurent plusieurs rôles physiologiques aux rang desquels la régulation et la régénération cellulaire (Auldridge et al., 2006). Selon la matrice de corrélation des paramètres lipidiques et leurs corollaires (Tableau 5), il existe une corrélation significative $(p<0,01)$ entre les indicateurs ou facteurs de risque (TG, C-LDL, VLDL) qui sont contrebalancés par le C-HDL. Selon une régression des moindres carrés entre les quantités de CT consommées et les RAC (risques d'attaque cardiaque) correspondants $\left(y=-5,762 x+10,40 ; R^{2}=\right.$ 0,950), la valeur du RAC est égale à l'unité lorsque l'animal consomme 1,63 à 1,81 g.MS.CT/100g.p.c/j.

\section{Enzymes et les organes}

Les trois transaminases sont couramment dosées comme des révélatrices de la toxicité d'un métabolite (Etuk et al., 2009a et 2009b). Selon Etuk et al. (2009a), les composés chimiques et les médicaments induisent la peroxydation lipidique, endommagent les membranes des cellules hépatiques et des organites, causent alors une inflammation et la nécrose des hépatocytes, d'où libération des transaminases cytosoliques tels que ASAT, ALAT, GGT, phosphatase alcaline dans la circulation sanguine. l'augmentation du taux sanguin de l'ASAT est associée à un infarctus du myocarde et à des lésions hépatiques, celle de l'ALAT à des lésions hépatiques ou celle de GGT à une cholestase ou des infarctus viscéraux (Quevauvilliers et al., 2007). Selon Akinloye et Ayankojo (2011), les degrés d'atteintes hépatiques sont exprimés par les ratios ASAT/ALAT $>1$ pour une cytolyse hépatique chronique ou sévère ASAT/ALAT $<1$ en cas de cytolyse hépatique moindre.

$\mathrm{Si}$ ces transaminases sont couramment dosées comme révélatrices de la toxicité d'un métabolite, dans le présent travail, le taux des $\gamma$-GT est inférieur à $5 \mathrm{UI} / \mathrm{L}$ chez tous les rats ayant consommé de $C$. tinctorium. Cela montre que les quantités consommées de $C$. tinctorium n'ont pas provoqué d'effets nécrotiques ou désordres au niveau des cellules des organes précités. De leur côté, Inngjerdingen et al. (2004) ont montré que l'administration d'extrait de $C$. tinctorium aux rats chez lesquels une insuffisance hépatique a été créée à l'aide de tétrachlorure de carbone a permis de guérir les lésions, justifiant ainsi l'effet hépatoprotecteur de $C$. tinctorium contre diverses intoxications observées (Akinloye et Ayankojo, 2011; Musa, 2012). On notera alors que la masse consommée par chaque groupe de rats équivalait à de principes actifs correspondants à de faibles doses stimulant la lipogenèse. C'est pourquoi le facteur de risque d'attaque cardiaque est inversement proportionnel à la quantité de $C$. tinctorium consommée.

Après dissection des rats, l'examen des organes n'a révélé aucune différence significative ( $>00,05)$ entre les 5 groupes. En raison de l'innocuité de $C$. tinctorium chez les rats, l'augmentation d'ALAT et d'ASAT se justifierait par une activité hépatique importante (Etuk et al., 2009 ; Musa, 2012).

\section{Effet général de $C$. tinctorium sur les rats}

En effet, d'après l'étude de corrélation des facteurs antinutritionnels de $C$. 
tinctorium à savoir les oxalates, les phytates, les tannins, les alcaloïdes, les flavonoïdes, avec les paramètres biochimiques (Tableau 6), la corrélation $\boldsymbol{r}$ a été significative $(\mathrm{p}<0,05$; $\mathrm{n}=5)$ mais négative entre tous les antinutriments et le gain de poids $(\mathrm{r}=-0,882)$. Aussi, la valeur de la corrélation a été négative mais non significative entre tous les antinutriments et la glycémie, le taux des protéines plasmatiques, les cendres fécales, la prise alimentaire, ou le cholestérol total. Toutefois, l'hypoglycémie a été positivement corrélée à une hypocholestérolémie $(\mathrm{r}=0,893)$.

Par contre, la corrélation $\boldsymbol{r}$ a été significative $(\mathrm{p}<0,05 ; \mathrm{n}=5)$ mais positive entre tous les antinutriments et les lipides fécaux $(r=0,902)$. Par ailleurs, les corrélations ont été positives entre tous les antinutriments et le taux des protéines fécales, entre antinutriments et $\mathrm{C}$-LDL, et dans une moindre mesure entre antinutriments et le cholestérol HDL ou entre antinutriments et C-VLDL/TG. De ce fait, l'incidence globale de ces corrélations positives a été observée sur la glycémie $(\mathrm{r}=-0,700)$ et le gain de poids $(\mathrm{r}=-$ 0,877 ) dont les valeurs ont été faibles par rapport aux groupes témoin ou porteur. La chute de la glycémie s'explique donc par le fait que le glucose soit désormais la seule source d'énergie utilisable par le jeune rat du fait, d'un côté, de la chute du taux de protéines plasmatiques en raison de l'augmentation du taux des protéines fécales, et de l'autre, par un rejet des lipides dans les fèces (par rapport au témoin). L'élimination des deux énergies métabolisables, protéines et lipides, a conduit à une chute de la glycémie chez les rats consommateurs de $C$. tinctorium par rapport au groupe témoin. Ici, il y a prédominance de synthèse du C-LDL sur la fraction C-HDL avec tendance à la baisse du cholestérol total $(\mathrm{r}=-0,692)$. Dans l'ensemble, la tendance à l'accumulation des triglycérides en rapport avec la consommation de $C$. tinctorium $(\mathrm{r}=0,477)$ atteste de l'effet hyperlipidémiant de cette racine qui, de plus, provoque une prévalence de l'accumulation du cholestérol LDL ( $r=0,716)$ en cas de faible consommation. Aussi, l'élimination accrue des minéraux par les fèces s'accompagne d'une baisse de la teneur en triglycérides plasmatiques $(\mathrm{r}=-0,901)$. Ce phénomène s'expliquerait par la déperdition des minéraux activateurs des enzymes responsables de la synthèse des triglycérides chez l'animal; cette déperdition est due aux chélateurs des minéraux divalents tels que les phytates, les oxalates et les tannins (Ndouyang et al., 2015).

Ainsi, le cercle de corrélation du système d'axes F1 $(65,83 \%)$ et F2 $(18,83 \%)$, et le biplot (axes F1 et F2: 83,84 \%) démontrent la distribution des variables indépendantes (groupes des rats) et des variables dépendantes (paramètres biochimiques) (Figures 4 et 5). Il en ressort que, suivant l'axe F1 $(65,83 \%)$, le groupe témoin (sans apéritif) et le groupe porteur ou référence (manioc) ont été sujets à la prise de poids, mais avec une glycémie et un taux de protéines plasmatiques élevés par rapport aux groupes testés au C. tinctorium CT1, CT2 et CT3. Cette étude des paramètres biochimiques a montré des corrélations inverses entre les effets de consommation de $C$. tinctorium surtout quand on considère les paramètres lipidiques. 
Tableau 5: Matrice de corrélation des paramètres lipidiques des rats ayant consommé des appétitifs au C. tinctorium.

\begin{tabular}{|c|c|c|c|c|c|c|c|c|}
\hline Variables & C-total & C-HDL & C-LDL & C-VLDL & TG & TG/C-HDL & RAC & IA \\
\hline C-total & 1 & & & & & & & \\
\hline C-HDL & $-0,204$ & 1 & & & & & & \\
\hline C-LDL & $-0,468$ & $0,956^{*}$ & 1 & & & & & \\
\hline C-VLDL & $-0,365$ & $0,949 *$ & $0,940 *$ & 1 & & & & \\
\hline TG & $-0,365$ & $0,949 *$ & $0,940 *$ & 1 & 1 & & & \\
\hline TG/C-HDL & $-0,734$ & 0,767 & 0,878 & $0,894 *$ & 0,894* & 1 & & \\
\hline RAC & $-0,781$ & 0,685 & 0,815 & 0,848 & 0,848 & $0,991 *$ & 1 & \\
\hline IA & $-0,655$ & 0,868 & $0,959 *$ & $\mathbf{0 , 9 3 3} *$ & $0,933^{*}$ & $0,976 *$ & $0,943 *$ & 1 \\
\hline
\end{tabular}


Tableau 6: Corrélation entre les facteurs antinutritionnels de $C$. tinctorium et les paramètres biochimiques des rats.

\begin{tabular}{|c|c|c|c|c|c|c|c|c|c|c|c|c|c|c|c|c|c|c|}
\hline Variables & $\begin{array}{l}\text { Oxa- } \\
\text { lates }\end{array}$ & $\begin{array}{l}\text { Phy- } \\
\text { tates }\end{array}$ & $\begin{array}{l}\text { Tan- } \\
\text { nins }\end{array}$ & $\begin{array}{l}\text { Alca- } \\
\text { loïdes }\end{array}$ & $\begin{array}{l}\text { Flavo- } \\
\text { noïdes }\end{array}$ & $\begin{array}{c}\text { Gly- } \\
\text { cémie }\end{array}$ & $\begin{array}{c}\text { Prot. } \\
\text { plasma }\end{array}$ & $\begin{array}{l}\text { Lipides } \\
\text { fécales }\end{array}$ & $\begin{array}{c}\text { Protéines } \\
\text { fécales }\end{array}$ & $\begin{array}{l}\text { Cendres } \\
\text { fécales }\end{array}$ & $\begin{array}{c}\text { Prise } \\
\text { aliment }\end{array}$ & $\begin{array}{l}\text { Gain } \\
\text { Poids }\end{array}$ & $\begin{array}{c}\mathrm{C}- \\
\text { total }\end{array}$ & $\begin{array}{c}\text { C- } \\
\text { HDL }\end{array}$ & $\begin{array}{c}\text { C- } \\
\text { LDL }\end{array}$ & $\begin{array}{c}\text { C- } \\
\text { VLDL }\end{array}$ & TG & $\begin{array}{l}\text { Crois- } \\
\text { sance }\end{array}$ \\
\hline Oxal & 1 & & & & & & & & & & & & & & & & & \\
\hline Phytates & 1 & 1 & & & & & & & & & & & & & & & & \\
\hline Tannins & 1 & 1 & 1 & & & & & & & & & & & & & & & \\
\hline Alcaloïdes & 1 & 1 & 1 & 1 & & & & & & & & & & & & & & \\
\hline Flavonoïdes & 1 & 1 & 1 & 1 & 1 & & & & & & & & & & & & & \\
\hline Glycémie & $-0,700$ & $-0,700$ & $-0,700$ & $-0,700$ & $-0,700$ & 1 & & & & & & & & & & & & \\
\hline Prot. plasma & $-0,877$ & $-0,877$ & $-0,877$ & $-0,877$ & $-0,877$ & 0,872 & 1 & & & & & & & & & & & \\
\hline Lipides féc. & $0,902 *$ & $0,902 *$ & $0,902 *$ & $0,902 *$ & $0,902 *$ & $-0,616$ & $-0,779$ & 1 & & & & & & & & & & \\
\hline Protéines féc. & 0,675 & 0,675 & 0,675 & 0,675 & 0,675 & $-0,349$ & $-0,303$ & 0,596 & 1 & & & & & & & & & \\
\hline Cendres féc. & $-0,447$ & $-0,447$ & $-0,447$ & $-0,447$ & $-0,447$ & 0,465 & 0,718 & $-0,228$ & 0,273 & 1 & & & & & & & & \\
\hline Prise aliment & $-0,567$ & $-0,567$ & $-0,567$ & $-0,567$ & $-0,567$ & $-0,101$ & 0,311 & $-0,690$ & $-0,332$ & 0,123 & 1 & & & & & & & \\
\hline Gain Poids & $-0,882 *$ & $-0,882 *$ & $-0,882 *$ & $-0,882 *$ & $-0,882 *$ & 0,770 & $\mathbf{0 , 9 3 1}$ & $-0,932 *$ & $-0,357$ & 0,513 & 0,533 & 1 & & & & & & \\
\hline C-total & $-0,692$ & $-0,692$ & $-0,692$ & $-0,692$ & $-0,692$ & $\mathbf{0 , 8 9 3 *}$ & 0,838 & $-0,425$ & $-0,343$ & 0,651 & $-0,158$ & 0,606 & 1 & & & & & \\
\hline C-HDL & 0,514 & 0,514 & 0,514 & 0,514 & 0,514 & $-0,068$ & $-0,541$ & 0,438 & $-0,115$ & $-0,754$ & $-0,710$ & $-0,536$ & $-0,204$ & 1 & & & & \\
\hline C-LDL & 0,716 & 0,716 & 0,716 & 0,716 & 0,716 & $-0,340$ & $-0,752$ & 0,590 & 0,067 & $-0,827$ & $-0,651$ & $-0,706$ & $-0,468$ & $0,956 *$ & 1 & & & \\
\hline C-VLDL & 0,477 & 0,477 & 0,477 & 0,477 & 0,477 & $-0,253$ & $-0,652$ & 0,386 & $-0,273$ & $-0,901 *$ & $-0,497$ & $-0,588$ & $-0,365$ & $0,949 *$ & $0,940 *$ & 1 & & \\
\hline TG & 0,477 & 0,477 & 0,477 & 0,477 & 0,477 & $-0,253$ & $-0,652$ & 0,386 & $-0,273$ & $-0,901 *$ & $-0,497$ & $-0,588$ & $-0,365$ & $0,949 *$ & $0,940 *$ & $1 *$ & 1 & \\
\hline Croissance & 0,676 & 0,676 & 0,676 & 0,676 & 0,676 & 0,042 & $-0,284$ & 0,623 & 0,690 & $-0,033$ & $-0,851$ & $-0,400$ & $-0,049$ & 0,529 & 0,550 & 0,273 & 0,273 & 1 \\
\hline
\end{tabular}

Les valeurs avec astérisque sont significative $(\mathrm{p}<0,05 ; \mathrm{n}=5)$. 


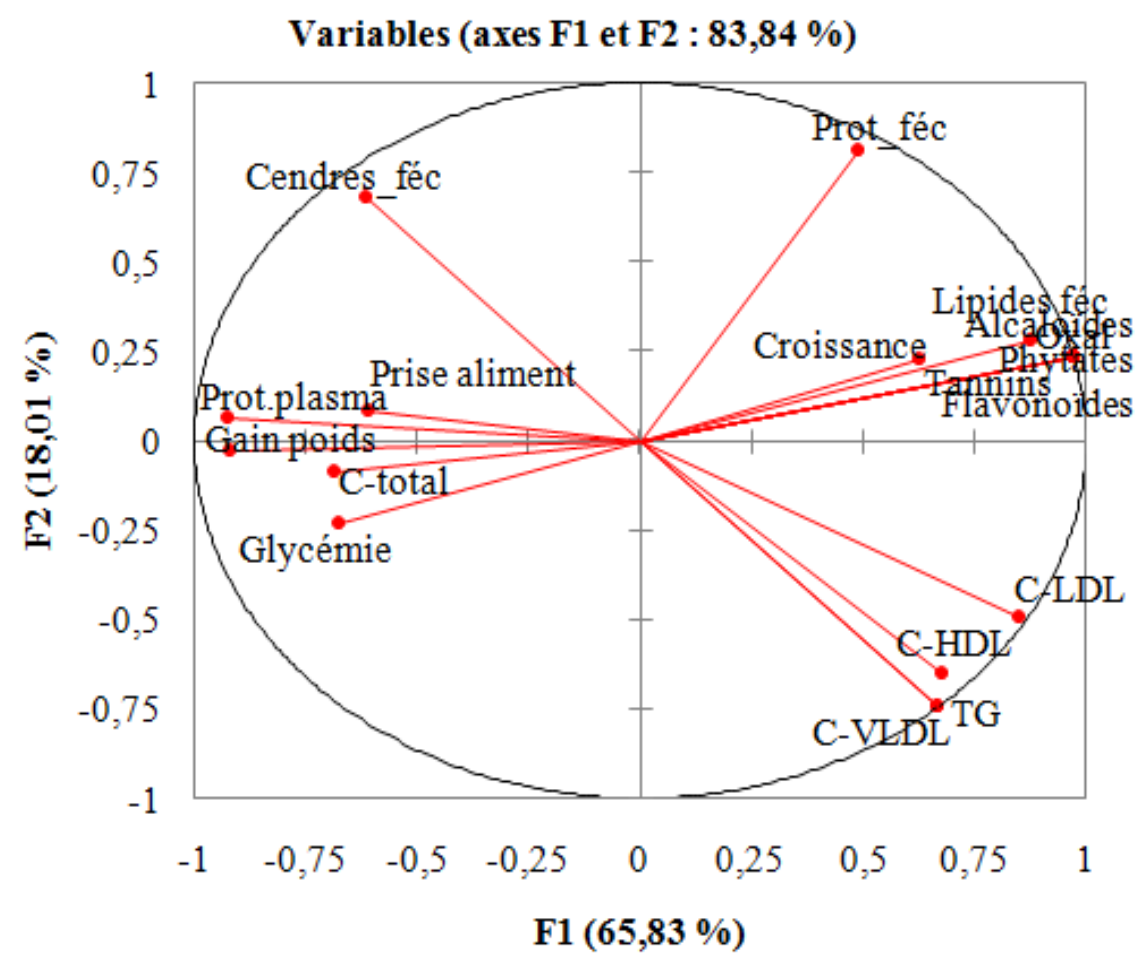

Figure 4: Cercle de corrélation entre les antinutriments de $C$. tinctorium et les paramètres nutritionnels.

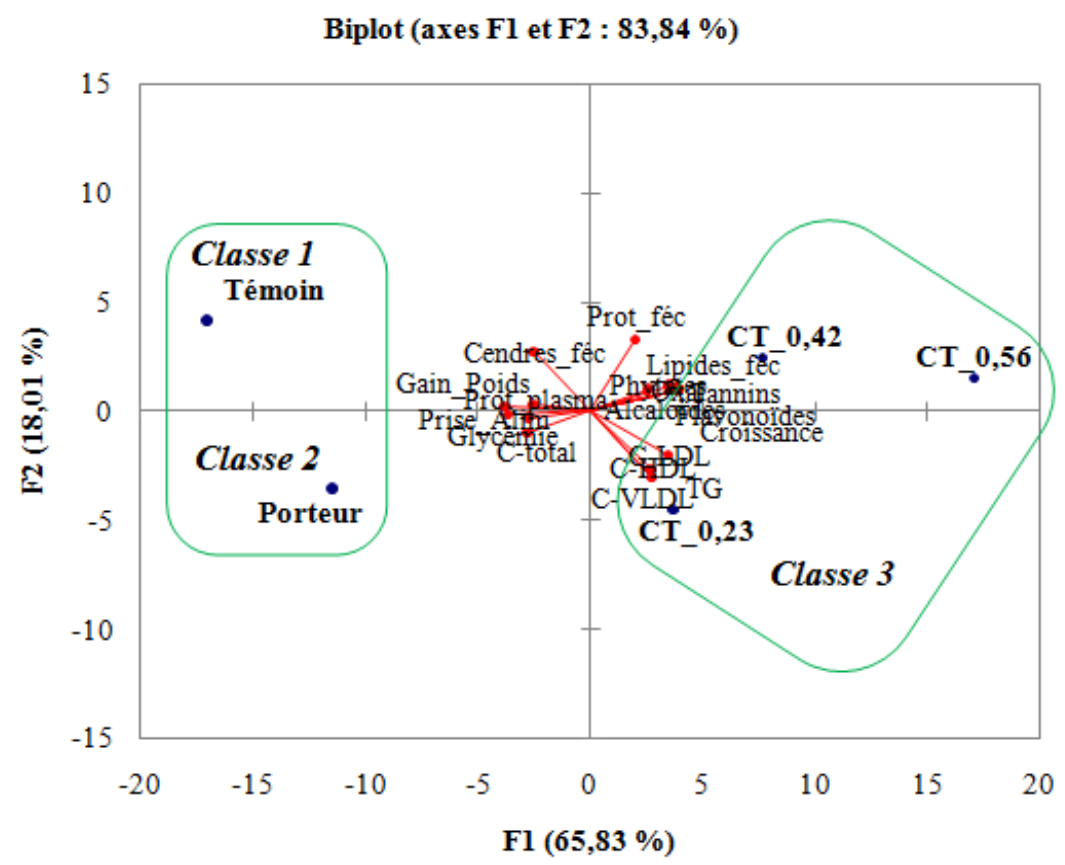

Figure 5: Biplot de corrélation entre les antinutriments de $C$. tinctorium et les paramètres nutritionnels. 


\section{Conclusion}

La farines de $C$. tinctorium affecte le staut nutritionnel du jeune rat selon la quantité consommée. Ainsi, la farine entière de $C$. tinctorium stimule chez le rat une accumulation des lipides mais dans une moindre mesure. Cette farine présente aussi des propriétés hypoglycémiantes et hypocholestérolémiantes. Dans ce sens, le statut nutritionnel du jeune rat dépend de la quantité consommée de la farine de $C$. tinctorium. Dans l'ensemble, la farine de $C$. tinctorium ralentit le gain de poids chez le jeune rat mais sans effet significatif sur la croissance. En outre, de indices de toxicité n'ont pas pu être démontrés chez le rat consommateur de C. tinctorium. En fait, l'emploi de cette plante comme aliment ou ingrédient culinaire dépend de l'usage dont on veut en faire en raison des fortes teneurs en composés phénoliques (tannins) et phytates chez $C$. tinctorium. Finalement, la poursuite de l'étude fonctionnelle des poudres de $C$. tinctorium apparaît nécessaire pour faciliter leur usages pratiques.

\section{CONFLIT D'INTERETS}

Les auteurs de la présente étude déclarent qu'il n'y a aucun conflit d'intérêts entre eux.

\section{CONTRIBUTIONS DES AUTEURS}

Dans la réalisation du présent travail, CJN a élaboré le protocole de recherche, collecté et traité les données et aussi rédigé le manuscrit ; HM a participé à la relecture du document ; CJN et RMN ont effectué le traitement des données.

\section{REMERCIEMENTS}

Les auteurs de cet article remercient l'ENSAI de l'Université de Ngaoundéré (Cameroun) pour la disponibilité de ses laboratoires spécialisés qui ont permis la réalisation de ce travail multidisciplinaire.

\section{REFERENCES}

Abu AH, Ochalefu DO, Ibrahim A. 2012. Aqueous ethanolic extract of
Cochlospermum planchonii rhizome enhances spermatogenesis in male albino rats. African Journal of Biotechnology, 11(53): $\quad$ 11636-11639. DOI 10.5897//AJB12.225.

Ahmed TS, Magaji MG, Yaro AH, Musa AM, Adamu AK. 2011. Aqueous methanol extracts of Cochlospermum tinctorium (A. Rich) possess analgesic and antiinflammatory activities. Pharmacology, : 3(3) : 237-242. DOI: 10.4103/09751483.83774 .

Akinloye OA, Ayankojo AG 2011. Hepatoprotective activity of Cochlospermum tinctorium against carbon tetrachloride induced hepatotoxicity in rats. Agriculture and Biology Journal of North America, 2(9): $\quad 1283-1288$. Doi:10.5251/abjna.2011.2.9.1283.1288.

Auldridge Michele E, McCarty DR, Klee HJ. 2006. Plant carotenoid cleavage oxygenases and their apocarotenoid products. Current Opinion in Plant Biology, 9: 315-321. DOI 10.1016/j.pbi.2006.03.005.

Bayaga HN, Guedje NM, Biye EH. 2017. Approche ethnobotanique et ethnopharmacologique des plantes utilisées dans le traitement traditionnel de l'ulcère de Buruli à Akonolinga (Cameroun). Int. J. Biol. Chem. Sci., 11(4): 1523-1541. DOI: https://dx.doi.org/10.4314/ijbcs.v11i4.10

Dada AA. 2012. Effects of herbal growth promoter feed additive in fish meal on the performance of Nile Tilapia (Oreochromis niloticus (L.)). Egypt. Acad. J. Biolog. Sci., 4(1): 111-117. DOI: $\quad$ zoology.eajbs.eg.net/pdf/vol4num1/12.

De Almeida SCX, De Lemos TLG, Silveira ER, Pessoa ODL. 2005. Constituintes químicos voláteis e não-voláteis de Cochlospermum vitifolium (Willdenow) Sprengel. Quim. Nova, 28(1): 57-60. DOI : http://dx.doi.org/10.1590/S010040422005000100012. 
Etuk EU, Agaie BM, Ladan MJ, Garba I. 2009a. The modulatory effect of Cochlospermum tinctorium a rich aqueous root extract on liver damage induced by carbon tetrachloride in rats. African Journal of Pharmacy and Pharmacology, 3(4): 151-157. DOI: http://www.academicjournals.org/ajpparticle1380876644.

Etuk EU, Francis UU, Garba I. 2009 b. Regenerative action of Cochlospermum tinctorium aqueous root extract on experimentally induced hepatic damage in rats. African Journal of Biochemistry Research 3(1): 001-004. DOI: http://www.academicjournals.org/AJBR/ / F9A7D9B10319.

Feng QL, Wu J, Chen GQ, Cui FZ, Kim TN, Kim JO. 2000. A mechanistic study of the antibacterial effect of silver ions on Escherichia coli and Staphylococcus aureus. J. Biomed. Mater. Res., 52: 662. DOI: www.hydrosolinfo.com/pdf/3.pdf.

Gao Y, Shang C, Saghai Maroof MA, Biyashev RM, Grabau EA, Kwanyuen P, Burton JW, Buss GR. 2007. A Modified Colorimetric Method for Phytic Acid Analysis in Soybean. Crop Science, 47(5): 1797-1803. DOI : 10.2135/cropsci2007.03.0122.

Gueye M, Cissé A, Diatta CD, Diop S, Koma S. 2012. Etude ethnobotanique des plantes utilisées contre la constipation chez les Malinké de la communauté rurale de Tomboronkoto, Kédougou (Sénégal). Int. J. Biol. Chem. Sci., 6(2): 773-781.

DOI: http://dx.doi.org/10.4314/ijbcs.v6i2.19.

ICON. 2008. Towards Predicting NanoBiointeractions: An International Assessment of Nanotechnology Environment, Health and Safety Research Needs. International Council on Nanotechnology, 4(1): 80. URL: $\mathrm{http}: / /$ icon.rice.edu/projects.cfm?doc_id= 12220

Inngjerdingen K, Nergard CS, Diallo D, Mounkoro PP, Paulsen BS. 2004. An Ethnopharmacological survey of plants used for wound healing in Dogonland, Mali, West Africa. Journal of ethnopharmac ology, 92: 233-244. Doi: 10.1016/j.jep.2004.02.021.

Jeppesen J, Hein HO, Suadicani P, Gyntelberg F. 2001. Low triglycerides-high highdensity lipoprotein cholesterol and risk of ischemic heart disease. Arch Intern Med.,161: 361-366.

Kouadio KKA, Coulibaly S, Atchibri LO-A, Kouamé G, Meité A. 2012. Evaluation nutritionnelle comparative des fruits de trois hybrides de bananiers (CRBP 39, FHIA 17 et FHIA 21) avec ceux de la variété Orishele. Tropicultura, 30(1): 4954.

DOI: www.tropicultura.org/text/v30n1/49.pdf

Kouakou R, Kouassi AM, Kwa-Koffi EK, Gnonsoro UP, Trokourey A. 2015. Distribution of Polycyclic Aromatic Hydrocarbons (PAHs) in a tropical coastal lagoon (Grand-Lahou lagoon, Côte d'Ivoire). Int. J. Biol. Chem. Sci., 9(2): 2020-2029. DOI: http://dx.doi.org/10.4314/ijbcs.v9i2.47.

Makkar HPS, Siddhuraju P, Becker K. 2007. Plant Secondary Metabolites. Methods in Molecular Biology, 393: 128 p.

Morou B, Ounani H, Amadou OA, Diouf A, Guero C, Mahamane A. 2016. Caractérisation de la structure démographique des ligneux dans les parcs agroforestiers du terroir de Dan Saga (Aguié, Niger). Int. J. Biol. Chem. Sci., 10(3): 1295-1311. DOI: http://dx.doi.org/10.4314/ijbcs.v10i3.31.

Muanda FN, Bouayed J, Djilani A, Yao C, Soulimani R, Dicko A. 2011. Chemical composition and, cellular evaluation of the antioxidant activity of Desmodium adscendens leaves. Evidence-Based Complementary and Alternative Medicine, 2011:

Doi:10.1155/2011/620862.

Musa AA. 2012. Cytotoxicity Activity and Phytochemical Screening of Cochlospermum tinctorium Perr Ex A. Rich rhizome. Journal of Applied 
Pharmaceutical Science, 02(07): 155159 . DOI: 10.7324/JAPS.2012.2723.

Ndouyang CJ, Nguimbou RM, Njintang YN, Scher J, Facho B, Mbofung CMF. 2014. In Vivo Assessment of the Nutritional and Subchronic Toxicity of Tacca leontopetaloides (L.) Tubers. Scholarly Journal of Agricultural Science, 4(1):513. DOI: Doi: https://hal.archivesouvertes.fr/hal-01275339.

Ndouyang CJ, Njintang YN, Facho B, Scher J, Mbofung CMF. 2015. Effect of Processing Method on the Antinutrient Content of Tacca leontopetaloides (L.) Kuntze Flour. British Journal of Applied Science \& Technology, 5(3): 258-269. DOI: $10.9734 / B J A S T / 2015 / 10217$.

Ortiz-Andrade R, Torres-Piedra M, SanchezSalgado JC, Garcia-Ji-Menez S, Villalobos-Molinab R, Ibarra-Barajas M, Gallardo-Ortiz I and Estrada-Soto S. 2009. Acute and sub-chronic effects of Cochlospermum vitifolium in blood glucose levels in normoglycemic and STZ-nicotinamide-induced diabetic rats. Rev. Latinoamer. Quím., 37(2): 122-132. DOI:

https://www.researchgate.net/.../2022883 22.

Quevauvilliers J, Somogyi A, Fingerhut A. 2007. Dictionnaire Médical. Eds Masson : Paris ; 1093.

Sasikala A Savithramma N. 2012. Biological Synthesis of Silver Nanoparticles from Cochlospermum religiosum and their Antibacterial Efficacy. J. Pharm. Sci. \& Res., 4(6): 1836-1839. DOI citeseerx.ist.psu.edu/viewdoc/download? doi=10.1.1.295.6174.
Siddiq M, Rav R, Harte JB Dolan KD. 2010. Physical and functional characteristics of selected dry bean (Phaseolus vulgaricus (L.) flours. LWT-J. Fd. Sci. Tech., 43: 232-237. DOI: https://doi.org/10.1016/ j.lwt.2009.07.009

Solon S, Carollo CA Brandão LFG. 2012. Phenolic derivatives and other chemical compounds from Cochlospermum regium. Quim. Nova, 35(6): 1169-1172. DOI: http://dx.doi.org/10.1590/S010040422012000600019.

Song K-S, Shim J-Y, Jung D-S, Kim S-U. 2011. Origin of oxygen in indoxylderivatives of Polygonum tinctorium L. as probed by ${ }^{18} \mathrm{O}_{2}$ Feeding. J. Korean Soc. Appl. Biol. Chem., 54(3): 340-344. doi:10.3839/jksabc.2011.054.

Willcox M, Benoit-Vical F, Fowler D, Bourdy G, Burford G, Giani S, Graziose R, Houghton P, Randrianarivelojosia M, Rasoanaivo P. 2011. Do ethnobotanical and laboratory data predict clinical safety and efficacy of anti-malarial plants? Malaria Journal, 10(1): S1-S7. Doi; http://www.malariajournal.com/content/ 10/S1/S7.

Yakubu MT, Akanji MA, Nafiu MO. 2010. Anti-diabetic activity of aqueous extract of Cochlospermum planchonii root in alloxan-induced diabetic rats. Cameroon Journal of Experimental Biology, 6(2): 91-100. DOI: http://dx.doi.org/10.4314/ cajeb.v6i2.68526.

Zhou J, Qu F. 2011. Analysis of the extracts of Isatis tinctoria by new analytical approaches of HPLC, MS and NMR. Afr $J$ Tradit Complement Altern Med.; 8(5): 33-45. DOI: 10.4314/ajtcam.v8i5S.13. 